\title{
Effect of histone deacetylase inhibitors on cell apoptosis and expression of the tumor suppressor genes RUNX3 and ARHI in ovarian tumors
}

\author{
LAN ZHANG ${ }^{1,2}$, PEISHU LIU ${ }^{1}, \mathrm{HUA} \mathrm{LI}^{2}$ and FUYING XUE ${ }^{2}$ \\ ${ }^{1}$ Department of Obstetrics and Gynecology, Qilu Hospital, Shandong University, Ji'nan, Shandong 250000; \\ ${ }^{2}$ Department of Obstetrics and Gynecology, Tai'an Central Hospital, Tai'an, Shandong 271000, P.R. China
}

Received November 29, 2012; Accepted March 8, 2013

DOI: $10.3892 / \mathrm{mmr} .2013 .1371$

\begin{abstract}
The aim of this study was to investigate the expression of Runt box 3 (RUNX3) and aplasia Ras homolog member I (ARHI) in ovarian tumors, and the effects of histone deacetylase inhibitors (HDACIs) on the expression of these genes and the growth of ovarian cancer cells. The mRNA expression of the RUNX3 and ARHI genes in normal ovaries and ovarian tumors was determined using reverse transcription polymerase chain reaction (RT-PCR). The effects of HDACIs on RUNX3 and ARHI expression in four ovarian cancer cell lines (SKOV3, A2780, COC1 and OC3) were determined using RT-PCR and the MTT assay. The expression of RUNX3 and ARHI in normal ovarian cells was 86 and $100 \%$, respectively. Although the two genes were downregulated in ovarian tumors, the extent of downregulation differed. The expression of RUNX3 and ARHI was correlated with the degree of tumor malignancy $(\mathrm{P}<0.05)$. ARHI was expressed in all four ovarian cancer cell lines, whereas RUNX3 was expressed only in the OC3 cell line. Treatment with HDACIs upregulated the expression of ARHI and RUNX3 in the SKOV3 cell line only. In A2780 cells, HDACIs upregulated ARHI expression only in the presence of trichostatin A (TSA) plus cisplatin. HDACIs induced significant apoptosis in ovarian cancer cells, which was inversely correlated with the concentration and duration of treatment $(\mathrm{P}<0.05)$. In conclusion, RUNX3 and ARHI were shown to be expressed in normal ovarian cells; however, their expression was downregulated or lost in ovarian tumor cells. The combined detection of ARHI and RUNX3 expression may offer improved prediction and monitoring of ovarian malignancies. HDACIs were revealed to inhibit the growth of ovarian tumor cells and may constitute a novel therapeutic option for ovarian tumors.
\end{abstract}

Correspondence to: Dr Peishu Liu, Department of Obstetrics and Gynecology, Qilu Hospital, Shandong University, Ji'nan, Shandong 250000, P.R. China

E-mail: peishuliu12@163.com

Key words: Runt box 3, aplasia Ras homolog member I, ovarian cancer, polymerase chain reaction, trichostatin A, cell line

\section{Introduction}

Mortality due to ovarian cancer is higher in the urban areas of China compared with the rural areas. Thus, the early diagnosis and treatment of ovarian cancer is a priority in China. Chemotherapy is the standard treatment for ovarian cancer; however, the resistance of cancer cells to chemotherapeutic drugs leading to relapsed and refractory disease is a major concern. The activation of cellular oncogenes and inactivation of tumor suppressor genes are the primary causes of cancer development; therefore, these are the main topics in tumor research (1-4).

The tumor suppressor gene Runt box 3 (RUNX3) belongs to the RUNX transcription factor family and is located on human chromosome 1 in the p36.1 region. It contains $\geq 5$ exons and is the downstream target of the transforming growth factor- $\beta$ (TGF- $\beta$ )-mediated signal transduction pathway (4). RUNX3 expression has been shown to be downregulated in various malignant tumors (5). The main mechanisms attributed to its downregulation are promoter methylation and loss of heterozygosity. Although the effect of promoter $\mathrm{CpG}$ island methylation on the expression of RUNX3 has previously been investigated, there are no studies on the effect of histone acetylation on the expression of RUNX3 (4,5). Multiple transcript variants encoding different isoforms have been identified for this gene, which has been shown to interact with other transcription factors. Aplasia Ras homolog member I (ARHI), another tumor suppressor gene, is a Ras-related small G-protein with a novel N-terminal extension that inhibits the growth of ovarian and breast tumor cells $(1,2)$. It is located on chromosome 1 in the $\mathrm{p} 31$ region (3). The increased expression of transcription inhibition factors and polyadenylation of histone deacetylases (HDACs) have been shown to affect the expression of ARHI in tumors (1-5).

HDAC inhibitors (HDACIs) are a new class of chemotherapeutic drug that effectively inhibit histone deacetylase activity and simultaneously promote histone and non-histone protein acetylation (4,6-8). HDACIs regulate the expression and degradation of tumor targeting and apoptosis-related proteins. HDACIs also activate apoptotic signaling pathways at the transcriptional and post-translational levels. Therefore, HDACIs are capable of inducing the apoptosis of tumor cells. HDACs, in association with transcription factors E2F1 and E2F4 (as 
complexes), are important in downregulating ARHI expression. Although previous studies have demonstrated a low expression of ARHI in ovarian tumors, the association of ARHI expression with tumor staging and histological typing has not been previously investigated.

In the present study, the expression of the tumor suppressor genes RUNX3 and ARHI in ovarian tumor tissues was investigated, and the association between the occurrence and development of ovarian malignant tumors was determined. The two genes are located on the same chromosome within the same sites. We aimed to investigate whether they exhibit a synergistic effect in ovarian tumor suppression. To assess the effect of HDACIs on ovarian cancer cell growth, RUNX3 and ARHI expression in ovarian cancer cell lines treated with the HDACI and trichostatin A (TSA) $(5,9,10)$, together with cisplatin as a positive control was determined.

\section{Material and methods}

Clinical samples. Following a review of the inpatient registry between 2008 and 2011, fresh ovarian tissue specimens from 77 subjects (age range, 17-89 years; mean age, 42 years) were obtained from four different locations; the Shandong Provincial Hospital (Ji'nan, China), the Qilu Hospital (Ji'nan, China), the Ji'nan Central Hospital (Ji'nan, China) and the Tai'an Central Hospital (Tai'an, China). Among these, there were 15 normal ovarian tissue specimens, 13 cases of ovarian benign tumors (serous cystadenoma, 7 cases; mucinous cystadenoma, 2 cases; follicular cyst, 1 case; ovarian endometriosis cyst, 1 case; theca cell tumor, 1 case; benign teratoma, 1 case), 6 cases of borderline ovarian tumors (serous, 3 cases; mucinous, 1 case; Brenner tumor, 2 cases) and 43 cases of malignant ovarian tumors. Clinicopathological staging of the tumors was performed according to the International Federation of Gynecology and Obstetrics (FIGO) classification (11). The histological type and degree of differentiation of the tumors were determined as previously described (11). The obtained specimens were stored in a refrigerator at $-80^{\circ} \mathrm{C}$ until RNA extraction. The study was approved by the ethics committee of Tai'an Central Hospital, Tai'an, China, and written informed consent was obtained from all patients.

Cell lines and culture reagents. The human ovarian carcinoma cell line COC1 was purchased from the China Center for Type Culture Collection (CCTCC) of Wuhan University (Wuhan, China). OC3/taxol, SKOV3 and A2780 cell lines were from Department of Qilu Hospital laboratory. The Rose Park Memorial Institute (RPMI)-1640 culture medium was purchased from Invitrogen (Carlsbad, CA, USA) and calf serum was from the Hangzhou Sijiqing Biological Engineering Materials, Co., Ltd. (Hangzhou, China). The RNA extraction kit and MTT were from Shandong Aibo Technology \& Trading Co. Ltd. (Ji'nan, China). The polymerase chain reaction (PCR) kit was from Finnzymes (Espoo, Finland). TSA was purchased from Cayman Chemical Co. (Ann Arbor, MI, USA). The genes of interest, ARHI and RUNX3, and $\beta$-actin were synthesized by Shenergy Co., Ltd. (Shanghai, China).

Cell culture. All the ovarian cancer cell lines (OC3/taxol, SKOV3, A2780 and COC1) used in this study were obtained from highly malignant ovarian tumor cells, including serous and mucinous types, which are internationally accepted representative ovarian cancer cell lines. OC3/taxol, SKOV3 and A2780 cells were grown in adherent cultures and COC1 cells were grown in a suspension culture. All the cell lines were cultured in RPMI-1640 supplemented with $10 \%$ calf serum, $100 \mathrm{U} / \mathrm{ml}$ penicillin and $0.1 \mathrm{mg} / \mathrm{ml}$ streptomycin. Cell culture maintenance and drug intervention experiments were performed at $37^{\circ} \mathrm{C}$ in an incubator with $5 \% \mathrm{CO}_{2}$ under saturated humidity.

Determination of cell growth inhibition. When the cultures reached an exponential growth phase, the adherent cells were digested with trypsin and made into single-cell suspensions. The cell suspensions were centrifuged and the cell concentration was adjusted to $5 \times 10^{4}$ cells $/ \mathrm{ml}$. The cells were then seeded in 96-well plates which were purchased from Yubo Biological Engineering Materials, Co., Ltd (Shanghai, China) (100 $\mu \mathrm{l}$ of cell suspension/well) and after $24 \mathrm{~h}$, the cells were either treated with cisplatin or TSA (drug-treated group) or left untreated (control group). A blank control was also prepared with cell culture medium alone. TSA was used at concentrations of 100, 200, 300 and $400 \mathrm{nmol} / \mathrm{l}$, and cisplatin at concentrations of 10, $20,40,80$ and $200 \mu \mathrm{mol} / 1$. Following 24 and $48 \mathrm{~h}$ of incubation with the drug, the culture plates were centrifuged to obtain the supernatants. RPMI-1640 (Invitrogen) and MTT ((Shandong Biological Engineering Materials, Co., Ltd, Jinan, China) solution were added to the supernatants and these were incubated for $4 \mathrm{~h}$ under the same conditions. The culture fluid was then extracted and treated with dimethyl sulfoxide (DMSO) prior to low-speed oscillations of the solution for $10 \mathrm{~min}$. The absorbance of the samples was read at $492 \mathrm{~nm}$ using an ELISA plate reader (Matsu Yoshi Equipment Co. Ltd, Chengdu, China). A reference wavelength of $620 \mathrm{~nm}$ was used for background correction.

Reverse transcription (RT)-PCR. Approximately $100 \mathrm{mg}$ of tissue specimen was homogenized in guanidium thiocyanate (GIT) solution to lyse the cells. For RNA extraction from the cultured cells, drug-treated cells in the logarithmic growth phase were used. The total cellular RNA was extracted using $\mathrm{TRIzol}^{\mathrm{TM}}$ reagent, after which the concentration and purity of the extracted RNA were determined. First-strand cDNA synthesis was performed with the First-Strand RevertAid ${ }^{\mathrm{TM}}$ cDNA Synthesis kit (Invitrogen), using $3 \mu \mathrm{l}$ of total RNA and oligo(dT) primers (Shanghai Shenergy Gambling company, Shanghai, China). The quantitative PCR reaction mixture included $2 \mu \mathrm{l}$ of cDNA and $48 \mu \mathrm{l}$ of reaction mix with Taq DNA polymerase and a pair of primers. The specific primer sequences used for ARHI were forward, 5'-TCTCTCCGAGCAGCGCA-3' and reverse, 5'-ATCTTCCTGTGGGGCTTGAAGG-3'. The PCR conditions were as follows: a total of 35 cycles, with each cycle consisting of denaturation at $95^{\circ} \mathrm{C}$ for $5 \mathrm{~min}$ and $95^{\circ} \mathrm{C}$ for $45 \mathrm{sec}$, annealing at $60^{\circ} \mathrm{C}$ for $45 \mathrm{sec}$ and elongation at $72^{\circ} \mathrm{C}$ for $45 \mathrm{sec}$, followed by extension at $72^{\circ} \mathrm{C}$ for $7 \mathrm{~min}$. The primer sequences used for RUNX3 were forward, 5'-TGGCAGGCAATGACGA-3' and reverse, 5'-TGGCAGGCAATGACGA-3'; the PCR conditions were as follows: a total of 35 cycles, with each cycle performed at $94^{\circ} \mathrm{C}$ for $1 \mathrm{~min}, 56^{\circ} \mathrm{C}$ for $45 \mathrm{sec}$ and $72^{\circ} \mathrm{C}$ for $1 \mathrm{~min}$. All the strips were analyzed and the relative content of ARHI and RUNX3 mRNA was calculated using an automatic gel electrophoresis 
software (Shanghai Jiapeng Technology Co., Ltd, Shanghai, China).

Statistical analysis. Analyses were performed using the Stata 8.0 software. The mean \pm standard deviation (SD) was used for descriptive analyses. The Kruskal-Wallis (KW) test and analysis of variance were used to assess the differences in means among the groups. Multiple comparisons between any two groups were performed using the Student-Newman-Keuls (SNK) test. $\mathrm{P}<0.05$ was considered to indicate a statistically significant difference.

\section{Results}

RUNX3 and ARHI are downregulated in ovarian tumor tissue The frequency of RUNX3 mRNA expression was $86 \%$ among the normal ovarian tissue specimens, with only 2 of the $15 \mathrm{spec}$ imens showing a gene deletion and an average expression level of $0.18 \pm 0.08 \mathrm{ng} / \mathrm{l}$. Furthermore, RUNX3 mRNA was expressed in $5 / 13$ benign tumor tissues, with an average expression level of $0.11 \pm 0.02 \mathrm{ng} / 1$, and in $2 / 6$ borderline tumor tissues (positive rate of expression, $33 \%$ ), with an average expression level of $0.07 \pm 0.0028 \mathrm{ng} / \mathrm{l}$. Among the malignant tumor tissues, the RUNX3 gene deletion rate was estimated to be $86 \%$ and ARHI gene expression was observed in only 6/43 malignant tumor tissues, with an average expression level of $0.01 \pm 0.0035 \mathrm{ng} / \mathrm{l}$.

ARHI mRNA was strongly expressed in the majority of normal ovarian tissues, with an average expression level of $0.23 \pm 0.02 \mathrm{ng} / \mathrm{l}$. ARHI mRNA expression was observed in $9 / 13$ benign tumor tissues; the positive rate of expression was $69 \%$ and the average expression level was $0.13 \pm 0.08 \mathrm{ng} / \mathrm{l}$. ARHI mRNA was also expressed in 3/6 borderline tumor tissues, with an average expression level of $0.08 \pm 0.00058 \mathrm{ng} / \mathrm{l}$, and in $17 / 43$ malignant tumor tissues, with a positive expression rate of $39.5 \%$ and an average expression level of $0.01 \pm 0.06 \mathrm{ng} / \mathrm{l}$.

The mRNA expression of RUNX3 and ARHI in three types of tumor tissues was significantly lower compared with that in normal ovarian tissues $(\mathrm{P}<0.01)$. RUNX3 mRNA expression was significantly higher in benign tumors when compared with malignant tumors $(\mathrm{P}<0.05)$. The differences in ARHI mRNA expression between benign and borderline tumors, and borderline and malignant tumors were not statistically significant $(\mathrm{P}>0.05)$. However, the differences in RUNX3 mRNA expression between borderline and malignant tumors, and benign and malignant tumors were significant $(\mathrm{P}<0.05)$.

Association of mRNA expression with clinicopathological characteristics of malignant ovarian tumors. In the present study, ovarian tumor tissues were categorized according to histological type. The results showed that the positive rate of ARHI gene expression in ovarian endometrioid carcinoma (22.2\%) was lower compared with the serous (43.7\%), mucinous (50\%) and other types (33\%) of tumors. However, there was no significant difference in RUNX3 mRNA expression among the four histological tumor types ( $\mathrm{P}>0.05)$. ARHI mRNA expression levels were negatively associated with ovarian cancer clinicopathological staging $(\mathrm{P}<0.01)$. Multiple comparison tests showed that the difference in ARHI mRNA expression between stage I and other cancer stages was not statistically significant $(\mathrm{P}>0.05)$. However, the differences in ARHI mRNA
Table I. Association of ARHI expression with clinicopathological characteristics in ovarian tumors.

\begin{tabular}{lrrrr}
\hline & \multicolumn{3}{c}{ ARHI expression } & \\
\cline { 2 - 3 } Characteristics & + & \pm & - & Total \\
\hline Histological type, $\mathrm{n}$ & & & & \\
Serous & 3 & 4 & 9 & 16 \\
Mucinous & 3 & 3 & 6 & 12 \\
Endometrial & 0 & 2 & 7 & 9 \\
Other & 0 & 2 & 4 & 6 \\
Clinical stage, $\mathrm{n}$ & & & & \\
I & 1 & 2 & 2 & 5 \\
II & 2 & 4 & 3 & 9 \\
III & 3 & 5 & 17 & 25 \\
IV & 0 & 0 & 4 & 4 \\
\hline
\end{tabular}

+, strong expression; \pm , weak expression; -, no expression.

Table II. ARHI and RUNX3 expression in malignant ovarian tumors.

\begin{tabular}{lrrr}
\hline & \multicolumn{2}{c}{ ARHI expression (n) } \\
\cline { 2 - 3 } RUNX3 expression & + & - & Total (n) \\
\hline+ & 4 & 2 & 6 \\
- & 13 & 24 & 37 \\
Total & 17 & 26 & 43 \\
\hline
\end{tabular}

+ , strong expression; -, no expression.

expression between stages II, III and IV, and between stages III and IV were statistically significant $(\mathrm{P}<0.05)$.

RUNX3 mRNA expression among the four histological ovarian tumor types was not significantly different $(\mathrm{P}>0.05)$. Furthermore, RUNX3 mRNA levels were negatively associated with ovarian cancer clinicopathological staging $(\mathrm{P}<0.01)$. Finally, the difference in ARHI and RUNX3 mRNA expression was statistically significant in malignant tumors $(\mathrm{P}<0.05)$, but not in other histological types of tumors (Tables I and II).

Growth inhibitory effect of TSA on ovarian cancer cells. The growth inhibition rates of various concentrations of TSA and cisplatin for the four types of ovarian cancer cell lines used in this study are provided in Table III. As shown in Table III, various concentrations of TSA inhibit cell growth and the rate of cell growth inhibition increases with increasing TSA concentration $(\mathrm{P}<0.01)$. The growth inhibition rates were clearly increased with the application of $300 \mathrm{nmol} / 1 \mathrm{TSA}$. Furthermore, inhibition rates increased with longer treatment durations. The rate of TSA-mediated growth inhibition was not significantly different among the four cell types $(\mathrm{P}>0.05)$. Cisplatin also inhibited the growth of ovarian tumor cells; the growth inhibition rate and cisplatin concentration/duration of treatment were shown to be correlated $(\mathrm{P}<0.05)$. The growth 
Table III. Rate of cell growth inhibition caused by treatment with various concentrations of trichostatin A (TSA) and cisplatin in the four types of ovarian cancer cells.

\begin{tabular}{|c|c|c|c|c|c|c|c|c|c|c|}
\hline \multirow[b]{2}{*}{ Cells } & \multirow{2}{*}{$\begin{array}{l}\text { Duration of } \\
\text { treatment }(\mathrm{h})\end{array}$} & \multicolumn{4}{|c|}{ TSA concentration $(\mu \mathrm{mol} / \mathrm{l})$} & \multicolumn{5}{|c|}{ Cisplatin concentration $(\mu \mathrm{mol} / \mathrm{l})$} \\
\hline & & 100 & 200 & 300 & 400 & 10 & 20 & 40 & 80 & 200 \\
\hline \multirow[t]{2}{*}{ SKOV3 } & 24 & 0.276 & 0.367 & 0.532 & 0.621 & 0.166 & 0.320 & 0.345 & 0.698 & 0.857 \\
\hline & 48 & 0.461 & 0.636 & 0.785 & 0.869 & 0.238 & 0.362 & 0.408 & 0.774 & 0.890 \\
\hline \multirow[t]{2}{*}{ A 2780} & 24 & 0.251 & 0.332 & 0.557 & 0.689 & 0.145 & 0.277 & 0.321 & 0.624 & 0.807 \\
\hline & 48 & 0.365 & 0.584 & 0.721 & 0.806 & 0.223 & 0.398 & 0.434 & 0.792 & 0.842 \\
\hline \multirow[t]{2}{*}{$\mathrm{COC} 1$} & 24 & 0.310 & 0.461 & 0.710 & 0.758 & 0.162 & 0.287 & 0.316 & 0.607 & 0.784 \\
\hline & 48 & 0.394 & 0.547 & 0.668 & 0.814 & 0.235 & 0.334 & 0.410 & 0.684 & 0.801 \\
\hline \multirow[t]{2}{*}{ OC3 } & 24 & 0.221 & 0.304 & 0.446 & 0.537 & 0.066 & 0.287 & 0.346 & 0.368 & 0.477 \\
\hline & 48 & 0.279 & 0.425 & 0.587 & 0.694 & 0.202 & 0.321 & 0.349 & 0.462 & 0.622 \\
\hline
\end{tabular}

inhibition rate of OC3 cells was the lowest and there was no significant difference in the growth inhibition rates of the remaining three cell lines, SKOV3, A2780 and COC1 ( $\mathrm{P}>0.05)$. The TSA- and cisplatin-mediated inhibition of ovarian cancer cells was compared and the overall distribution of the differences was not identified to be statistically significant $(\mathrm{P}>0.05)$.

Re-expression of RUNX3 and ARHI is achieved in HDACI-treated ovarian cells. RUNX3 was minimally expressed in all the ovarian cancer cell lines tested, with the exception of the OC3 cell line. The ovarian cancer cell lines SKOV3, A2780, COC1 and OC 3 expressed endogenous ARHI in markedly small amounts and were used to assess the effect of HDACI-mediated ARHI re-expression in cell cultures. Treatment with $250 \mathrm{nmol} / 1 \mathrm{TSA}$ and $80 \mu \mathrm{mol} / 1$ cisplatin, which are close to their $50 \%$ inhibitory concentration $\left(\mathrm{IC}_{50}\right)$, led to significant changes in ARHI and RUNX3 expression in the four ovarian cancer cell lines; ARHI and RUNX3 were re-expressed in SKOV3 cells following TSA treatment. In A2780 and COC1 cells, neither TSA nor cisplatin affected the expression of ARHI. However, the re-expression of ARHI was detected when cells were treated with a combination of TSA and cisplatin, particularly in A2780 cells.

\section{Discussion}

Based on recent studies, the crude death rate due to ovarian cancer is $\sim 1.45 / 100,000$ cases, which is ranked 12 th of all the types of cancer and accounts for $1.45 \%$ of all the cancer sites in females $(1,8,10)$. A number of ovarian cancer patients were admitted to hospital and presented with a large pelvic mass, ascites and bowel complications. Although surgical resection is an option, it rarely has satisfactory results, which seriously affects patient survival. Thus, the early diagnosis and treatment of ovarian cancer are key factors in improving prognosis.

The expression of an imprinted gene is determined by the contributing parent. An imprinted gene does not obey Mendel's laws of inheritance, but relies on single-transfer phenomena of genetic traits; for example, certain genes show parent-of-origindependent monoallelic expression and certain alleles are weakly expressed or not expressed at all. The loss of heterozygosity or mutational inactivation leads to the loss of gene expression $(4,12$ 14). RUNX3 and ARHI are located on human chromosome 1 in the $1 \mathrm{p} 36.1$ and $1 \mathrm{p} 31$ regions, respectively. RUNX3 is a member of the RUNX3 family of Runt-related transcription factors and is also known as polyomavirus enhancer-core binding protein $2 /$ core binding factor (PEBP2/CBF). It is an important target of the TGF- $\beta$ signaling pathway. RUNX3 has been reported to be a tumor suppressor gene that is involved in the regulation of cell growth, development, apoptosis and cell signal transduction. Additional biological effects are known to be important in its transcriptional regulation. Examples of such mechanisms are $\mathrm{CpG}$ island methylation of its $\mathrm{P} 2$ promoter region and loss of heterozygosity, which lead to RUNX3 silencing. ARHI is a newly discovered paternal-derived imprinted tumor suppressor gene and is considered to be involved in cell cycle regulation and cell signal transduction, resulting in a negative regulatory effect on growth and development $(15,16)$.

In the present study, ARHI and RUNX3 were shown to be widely expressed in normal ovarian tissue, while they were markedly downregulated in ovarian tumors. Consequently, the loss of function of these genes is likely to be one of the most crucial events in the development of ovarian cancer. Our results suggest that the expression of tumor suppressor genes and tumor formation and progression are closely linked. The deletion rates of ARHI were 31 and $50 \%$ in benign and borderline tumor tissues, respectively; however, the deletion rate of ARHI was as high as $61 \%$ in malignant tumors. The deletion rate of RUNX3 was $14 \%$ in normal ovarian tissues; however, this was increased to $61 \%$ in benign and $86 \%$ in malignant tumors. Even in tumor tissues with positive RUNX3 expression, the average expression was lower compared with that in normal ovarian tissues. RUNX3 expression was detected in only 4/17 ARHI-positive malignant tumors. By contrast, ARHI expression was detected in 4/6 RUNX3-positive malignant tumors; significant differences were identified using single factor analysis of variance. Furthermore, we also compared ARHI and RUNX3 expression in different clinical stages and histological types of ovarian tumors. The expression of these tumor suppressor genes clearly decreased with the increasing degree of tumor malignancy. The various malignant tumors were compared based on their histological types and the deletion rate of RUNX3 in endometrioid 
carcinoma was the highest $(87.5 \%)$, which was significantly higher compared with the average rate of gene loss in other malignant tumors. This may be associated with the low volume in the endometrium. The present study further confirmed that the re-expression of ARHI or RUNX3 induced autophagy in breast or gastric cancer cells and enhanced the inhibitory effects of cisplatin. Thus, the activation of RUNX3 and ARHI activation may be used as a novel therapeutic strategy in ovarian cancer. In the present study, the effects of the loss of ARHI and RUNX3 expression in ovarian cancer tissues were investigated.

Multiple mechanisms may contribute to the downregulation of ARHI and RUNX3 expression (16-18). Histone acetylation is one of the important factors affecting this process. It has been reported that acetylation of histone H3 is significantly decreased in cancer epithelial cells compared with normal epithelial cells, which may affect the normal transcription process (14). According to a study by Weber et al (2), ARHI mRNA was strongly expressed in normal thyroid cells and weakly expressed in follicular thyroid cancer cells $(\mathrm{P}=0.0018)$; HDACIs were also shown to increase ARHI expression in FICB3 cells. TSA is a specific HDACI that not only activates the expression of ARHI, but also upregulates the expression of Von Hippel-Lindau and p53 genes. Furthermore, TSA downregulates the expression of the hypoxia-inducible factor 1 and vascular endothelial growth factor $(14,15)$. The results of the present study showed that the application of $300 \mathrm{nmol} / \mathrm{l} \mathrm{TSA}$ significantly inhibited the growth of all four ovarian cancer cell lines $(\mathrm{P}<0.01)$. Following treatment with an $\mathrm{IC}_{50}$ concentration of TSA (250 nmol/l) for $48 \mathrm{~h}$, ARHI mRNA expression in SKOV3 cells was significantly increased compared with that in the negative control $(\mathrm{P}<0.01)$, as indicated by RT-PCR analysis. Cisplatin alone (concentration, $80 \mu \mathrm{m} / \mathrm{l}$ ) also had a significant inhibitory effect on the growth of ovarian tumors. However, the growth inhibition rate of cisplatin-treated OC3 cells was lower compared with that of the remaining three cell lines. Conversely, there was no significant difference in the growth inhibition rate of the four types of TSA-treated ovarian tumor cells. Thus, the present study demonstrated the growth inhibitory effect of TSA on drug-resistant ovarian tumor cells.

Aberrant DNA methylation and decreased histone acetylation lead to the abnormal expression of ARHI and RUNX3 in tumors. Previous studies have investigated demethylation inhibitors in different tumor tissues to restore ARHI and RUNX3 expression. The results showed that the rate of tumor growth was decreased and apoptosis was accelerated. Whether cancer cells die during apoptosis may depend on the levels of ARHI and RUNX3. This study revealed that the activation of these genes by TSA resulted in the increased apoptosis of ovarian tumor cells. A previous study demonstrated that vorinostat (SAHA) effectively inhibits the growth of human pancreatic cancer cells through the activation of the malignant phenotype-related genes caspase-9 and -3 , thus inducing apoptosis (17).

RUNX3 and ARHI are essential for the induction of ovarian cancer cell death. In the present study, the two tumor suppressor genes were re-expressed by TSA treatment and ovarian cancer cell growth was significantly inhibited; this may also be associated with the upregulation of additional silenced genes. This suggests that mechanisms other than the re-expression of ARHI and RUNX3 may also be involved in the growth inhibition of ovarian cancer cells. RUNX3 and ARHI have clear synergistic effects, since they are both tumor suppressor genes located on the same chromosomal region and are associated with the loss of heterozygosity. The combined detection of ARHI and RUNX3 along with additional relevant genes has the potential to be a key method for the screening and early diagnosis of ovarian cancer, which may ultimately improve clinical diagnosis and treatment. Additional studies are required to further elucidate the role of ARHI and RUNX3 in the development and progression of ovarian cancer. Future studies are likely to focus on gene transfection experiments in animals to further investigate the growth inhibition of ovarian cancer cells.

\section{References}

1. Rosen DG, Wang L, Jain AN, et al: Expression of the tumor suppressor gene ARHI in epithelial ovarian cancer is associated with increased expression of p21WAF1/CIP1 and prolonged progression-free survival. Clin Cancer Res 10: 6559-6566, 2004.

2. Weber F, Aldred MA, Morrison CD, et al: Silencing of the maternally imprinted tumor suppressor ARHI contributes to follicular thyroid carcinogenesis. J Clin Endocrinol Metab 90: $1149-1155,2005$

3. Bao JJ, Le XF, Wang RY, et al: Reexpression of the tumor suppressor gene ARHI induces apoptosis in ovarian and breast cancer cells through a caspase-independent calpain-dependent pathway. Cancer Res 62: 7264-7272, 2002.

4. Yang N, Zhang L, Zhang Y and Kazazian HH Jr: An important role for RUNX3 in human L1 transcription and retrotransposition. Nucleic Acids Res 31: 4929-4940, 2003.

5. Fukamachi H: Runx 3 controls growth and differentiation of gastric epithelial cells in mammals. Dev Growth Differ 48: 1-13, 2006.

6. Hisatomi H, Nagao K, Wakita K and Kohno N: ARHI/NOEY2 inactivation may be important in breast tumor pathogenesis. Oncology 62: 136-140, 2002.

7. Luo RZ, Peng H, Xu F, et al: Genomic structure and promoter characterization of an imprinted tumor suppressor gene ARHI. Biochim Biophys Acta 1519: 216-222, 2001.

8. Derrane M: Gene imprinting: making an impression on cancer research. J Natl Cancer Inst 91: 16-18, 1999.

9. Osaki M, Moriyama M, Adachi K, et al: Expression of RUNX3 protein in human gastric mucosa, intestinal metaplasia and carcinoma. Eur J Clin Invest 34: 605-612, 2004.

10. Holzer AK, Katano K, Klomp LW and Howell SB: Cisplatin rapidly down-regulates its own influx transporter hCTR1 in cultured human ovarian carcinoma cells. Clin Cancer Res 10: 6744-6749, 2004.

11. Steffensen KD, Waldstrøm M, Grove A, et al: Improved classification of epithelial ovarian cancer: results of 3 danish cohorts. Int J Gynecol Cancer. 21: 1592-1600, 2011.

12. Takai N, Kawamata N, Gui D, Said JW, Miyakawa I and Koeffler HP: Human ovarian carcinoma cells: histone deacetylase inhibitors exhibit antiproliferative activity and potently induce apoptosis. Cancer 101: 2760-2770, 2004.

13. Fujii S, Luo RZ, Yuan J, et al: Reactivation of the silenced and imprinted alleles of ARHI is associated with increased histone $\mathrm{H} 3$ acetylation and decreased histone $\mathrm{H} 3$ lysine 9 methylation. Hum Mol Genet 12: 1791-1800, 2003.

14. Li L, Shi H, Yiannoutsos C, Huang TH and Nephew KP: Epigenetic hypothesis tests for methylation and acetylation in a triple microarray system. J Comput Biol 12: 370-390, 2005.

15. Danaher RJ, Jacob RJ, Steiner MR, Allen WR, Hill JM and Miller CS: Histone deacetylase inhibitors induce reactivation of herpes simplex virus type 1 in a latency-associated transcript-independent manner in neuronal cells. J Neurovirol 11: 306-317, 2005

16. Woolf E, Xiao C, Fainaru O, et al: Runx3 and Runx1 are required for CD8 T cell development during thymopoiesis. Proc Natl Acad Sci USA 100: 7731-7736, 2003.

17. Bangsow C, Rubins N, Glusman G, et al: The RUNX3 gene - sequence, structure and regulated expression. Gene 279: 221-232, 2001.

18. Waki T, Tamura G, Sato M, Terashima M, Nishizuka S and Motoyama T: Promoter methylation status of DAP-kinase and RUNX3 genes in neoplastic and non-neoplastic gastric epithelia. Cancer Sci 94: 360-364, 2003. 